\title{
Fixed combinations in the management of hypertension: perspectives on lercanidipine - enalapril
}

\author{
Vivencio Barrios' \\ Carlos Escobar ${ }^{2}$ \\ Rocio Echarri ${ }^{3}$ \\ 'Department of Cardiology, Hospital \\ Ramón y Cajal, Madrid; '²Department \\ of Cardiology, Hospital Infanta Sofia, \\ Madrid, ${ }^{3}$ Department of Nephrology, \\ Hospital Infanta Sofia, Madrid
}

\begin{abstract}
Although achieving blood pressure (BP) control is critical to improve cardiovascular prognosis in hypertensive patients, many of them fail to achieve BP goals. The majority of hypertensive patients need more than one antihypertensive agent to attain BP targets. Combination therapy is required when monotherapy fails to attain BP objectives and as a first-line treatment in certain situations, such as markedly elevated BP values, when lower targets are required in high or very high cardiovascular risk patients. The advantages of combination therapy are well documented, with an increased antihypertensive efficacy as a result of the simultaneous inhibition of different mechanisms of action and with a lesser incidence of adverse events, because of the possible compensatory responses and the lower doses used. Calcium channel blockers are effective drugs in the treatment of hypertension. The efficacy of lercanidipine has been evaluated in several noncomparative and in comparative studies showing a great efficacy with a good tolerability. On the other hand, the inhibition of the renin-angiotensin system appears to be very beneficial in the treatment of patients with hypertension. Enalapril is an effective and well tolerated angiotensin converting enzyme inhibitor. Although there are several fixedcombination drugs, the combination lercanidipine plus enalapril appears to be one of the most promising therapies in the treatment of hypertension. The aim of this manuscript is to update the published data about the efficacy and safety of this fixed combination.
\end{abstract}

Keywords: angiotensin converting enzyme inhibitors, calcium channel blockers, combination therapy, hypertension, lercanidipine, enalapril

\section{Introduction}

Hypertension is a major risk factor for cardiovascular disease. It has been estimated that the prevalence of hypertension is about $29.3 \%$ in general population and $66.3 \%$ in elderly (Ong et al 2007; Wang et al 2007). Blood pressure (BP) control is crucial to improve cardiovascular prognosis in hypertensive patients (Mancia et al 2007). In the US, data from the National Health and Nutrition Examination Survey show that only about one third of American hypertensive patients are adequately controlled (Cheung et al 2006). Although BP control is still far from optimal, in recent years it has markedly improved; as an example, in Spain, BP control has increased from less than $20 \%$ to the current $40 \%$ (Barrios et al 2007a). However, this situation may worsen since the most recent recommendations have proposed more strict BP objectives for patients at high risk such as those with diabetes, or cardiovascular or renal disease in which BP goals should be $<130 / 80 \mathrm{mmHg}$ (Mancia et al 2007). This is clinically very relevant, first, because current evidence shows that nowadays the majority of patients daily attended by physicians, regardless they are specialists or general practitioners, are at high or very high risk, and second, because the prevalence of obesity and diabetes is likely to grow in the near future, this situation will worsen (Barrios et al 2007b; Ryden et al 2007). 
Figure 1 shows the different BP control rates according to different cardiovascular risk conditions provided from a recently published survey that enrolled a total of 12,954 patients with hypertension assisted daily in primary care setting (Barrios et al 2007c, d, e). As shown, when cardiovascular risk increases, a lower proportion of patients attain BP goals.

It has been reported that most patients with hypertension will need at least two antihypertensive drugs to achieve BP goals, mainly those patients at higher risk (Motwani 2002; Sica 2002; Barrios et al 2007b; Ryden et al 2007). A study that analyzed the number of antihypertensive drugs used in different clinical trials reported that the median of agents used for each patient was 2.8. Despite this, most of them did not achieve optimal BP objectives (Lazarus et al 1997; Estacio and Schrier 1998; Hansson et al 1998; Bakris et al 2000; Brenner et al 2001; Lewis et al 2001; UKPDS 38 2001; ALLHAT investigators 2002; Dahlöf et al 2005). Although there is a growing awareness about the need of using combinations to attain BP goals in most patients, mainly in those at higher risk, their use in clinical practice is still far from desirable (Figure 2) (Barrios et al 2007c, d, e; Mancia et al 2007).

\section{Rationale of combined therapy}

Classically, guidelines for the management of arterial hypertension recommended "start low, go slow". This meant that treatment should be initiated at low doses and with slow increases, in order to avoid side effects, but assumed incorrectly that in most patients with hypertension there was only one underlying cause. However, current evidence shows that hypertension is caused by interacting multifactorial mechanisms, and this is more significant in high-risk patients (Nesbitt 2007).

As a result, to best treat these patients, it is necessary to use combinations of drugs with different mechanisms of action. Combined therapy can block counter-regulatory mechanisms and potentiate the antihypertensive efficacy beyond the additive response of each drug alone (McInnes 2007). Several trials have shown that when the titration of medication is very gradual, patients are at risk of presenting cardiovascular outcomes before BP goals are obtained, especially in those with diabetes or previous cardiovascular disease (Hansson et al 1998; UKPDS 1998; Julius et al 2003; Nesbitt 2007). The advantages of combination therapy are well documented: 1) Increased antihypertensive efficacy as a result of combining different mechanisms of action; 2) A lesser incidence of adverse effects because of the lower doses used and the possible compensatory responses; 3) Since fixed low-dose combinations are available, the treatment simplification may optimize compliance and, secondarily, enhance BP control; and 4) Starting treatment with a two-drug combination may allow BP goals to be achieved earlier than with only one antihypertensive drug (Mancia et al 2007).

In fact, the JNC-7 report and the European guidelines for the management of arterial hypertension recommend a more aggressive approach in some patients (Chobanian et al 2003; Mancia et al 2007). The JNC-7 report suggests that it should be started from the beginning with two antihypertensive drugs

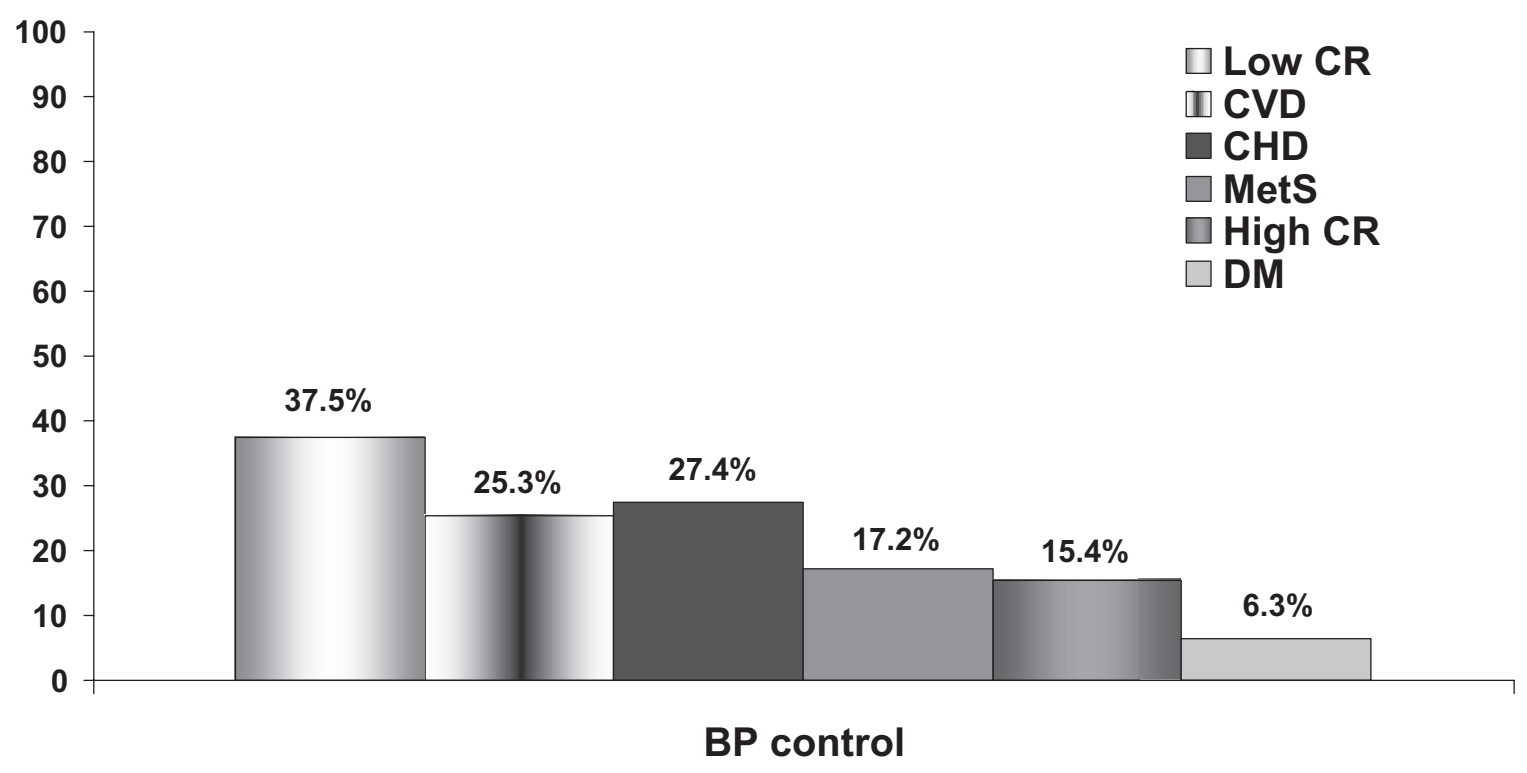

Figure I Blood pressure control rates according to different cardiovascular risk conditions (after Barrios et al 2007c, d, e). Abbreviations: CVD, cardiovascular disease; CHD, coronary heart disease; MetS, metabolic syndrome; CR, coronary risk; DM, diabetes mellitus. 


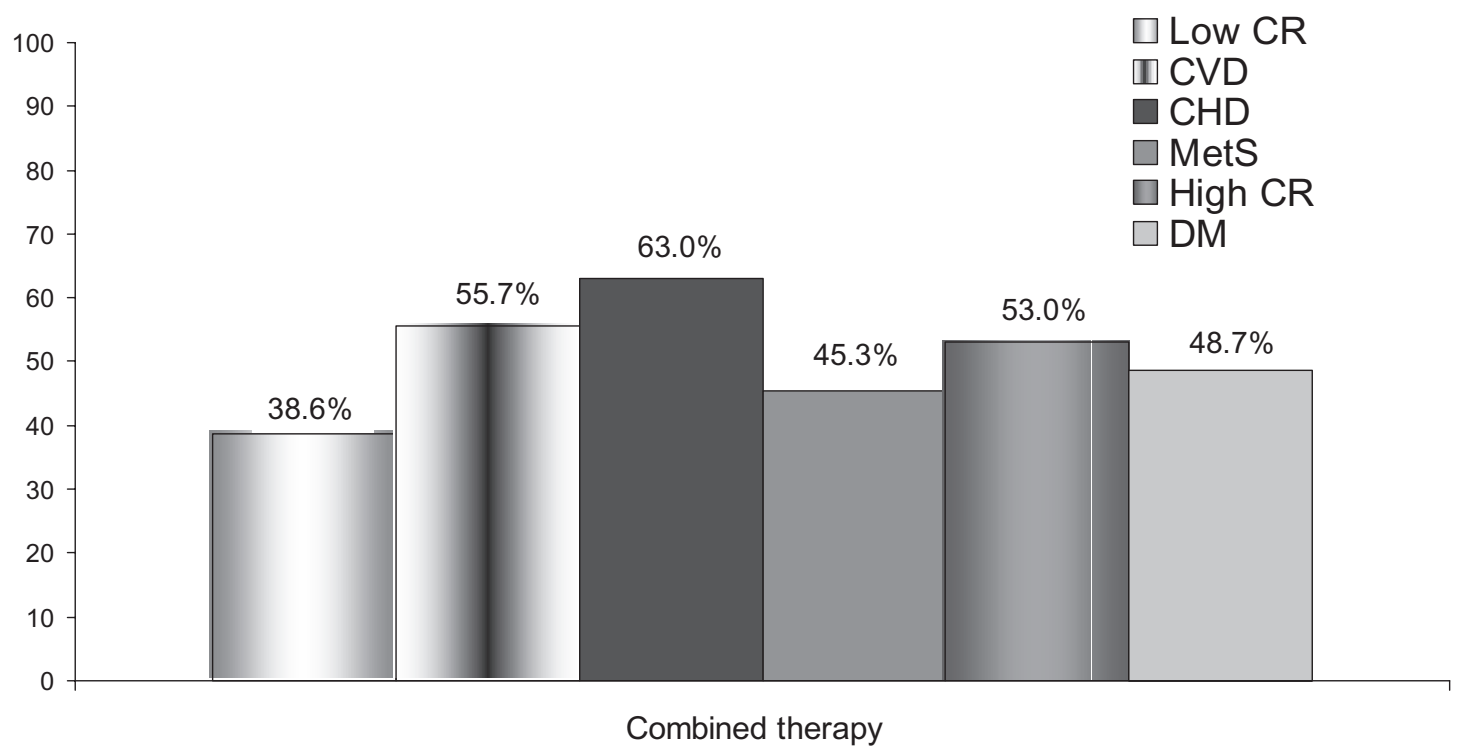

Figure 2 Use of combined therapy according to different cardiovascular risk conditions (after Barrios et al 2007c, d, e).

Abbreviations: CVD, cardiovascular disease; CHD, coronary heart disease; MetS, metabolic syndrome; CR, coronary risk; DM, diabetes mellitus.

in those patients with a systolic $\mathrm{BP}>20 \mathrm{mmHg}$ over $\mathrm{BP}$ goal and/or $>10 \mathrm{mmHg}$ over the diastolic goal (Chobanian et al 2003). European guidelines are more clear about this issue (Mancia et al 2007). Thus, as these recommendations suggest, combination therapy is necessary when monotherapy fails to attain BP objectives but also as first-line treatment in some situations, such as high or very high cardiovascular risk, when lower BP targets are required, or when BP elevations are marked (Mancia et al 2007).

In clinical practice there are numerous fixed-dose antihypertensive combination regimens: beta-blockers + hydrochlorothiazide (HCTZ), angiotensin converting enzyme inhibitors $(\mathrm{ACEi})+\mathrm{HCTZ}$, angiotensin receptor blockers $(\mathrm{ARB})+$ HCTZ, and calcium channel blockers (CCB) + ACEi (Barrios and Escobar 2008). One of the most promising combinations is lercanidipine (a CCB) + enalapril (an ACEi).

\section{Combining CCB and ACEi: beyond blood pressure control}

Several randomized clinical trials and studies performed in conditions of daily clinical practice have shown that CCBs are potent antihypertensive drugs with a good tolerability not only in the general hypertensive population but also in high risk patients, alone or in combination (Staessen et al 1997; Liu et al 1998; Barrios et al 2002, 2006; Nissen et al 2004; Hasebe and Kikuchi 2005; Egan 2007; Fogari et al 2007a, b, c; Otero 2007; Poldermans et al 2007; Sowers et al 2007).

Lercanidipine is a third-generation vasoselective dihydropyridine (DHP) acting through the blockade of the L-type calcium channels in cell membranes (Herbette et al 1998; Meredith 1999; Epstein 2001; Hair et al 2007). This drug has a high lipophilicity, which enables a slower and smooth onset and longer duration of action than other classic DHPs (Meredith 1999). Lercanidipine is highly selective for vascular smooth muscle because of the high proportion of L-type calcium channels in this tissue (Hair 2007). The efficacy of lercanidipine has been evaluated in non-comparative and in comparative studies with other CCBs and different antihypertensive drugs, showing comparable effects in all the cases (Barrios et al 2002, 2006; James et al 2002; Leonetti et al 2002; Cherubini et al 2003; Romito et al 2003). The effect of lercanidipine has also been successfully evaluated in severe or resistant hypertension, elderly subjects, and diabetics (Bang et al 2003; Agrawal et al 2006).

Moreover, some studies have suggested that lercanidipine may have anti-atherogenic effects beyond BP reductions (Soma et al 1998; Rachmani et al 2002; Canavesi et al 2004). As an example, lercanidipine reduced by $35 \%(\mathrm{p}<0.001)$ the levels of low-density lipoprotein cholesterol oxidation, measured as conjugated diene formation, in a hypertensive population with diabetes mellitus (Rachmani et al 2002). Other benefits reported with lercanidipine are their renoprotective effects and favorable effects on lipid profile and glucose tolerance (Dalla Vestra et al 2004; Robles et al 2005; Barrios et al 2008). Thus, a study that recruited 203 chronic renal failure patients (creatinine $>1.4 \mathrm{mg} / \mathrm{dL}$ for males, creatinine $>1.2 \mathrm{mg} / \mathrm{dL}$ for females, or creatinine clearance $<70 \mathrm{~mL} / \mathrm{min}$ ) showed that lercanidipine had a 
high antihypertensive effect with a good tolerability profile in patients with renal failure. Moreover, an improvement in renal function assessed by creatinine clearance was described (Robles et al 2005). On the other hand, lercanidipine has been shown to have neutral or favorable effects on lipid profile and to enhance glucose tolerance and to reduce fasting blood glucose and glycosylated haemoglobin in diabetics with hypertension (Bang et al 2003; Hair et al 2007). Lercanidipine is a well tolerated drug with a low adverse events rate due to its long-lasting and vascular-selective calcium entryblocking activity, while sympathetic activation and reflex tachycardia is not induced (Epstein 2001). As a result, the overall adverse events rate is lower than that observed with other DHPs (Cherubini et al 2003; Barrios et al 2008).

The renin-angiotensin-aldosterone system (RAAS) plays a key role in regulating blood volume and systemic vascular resistance. The over-activation of this system, especially through the excessive production of its effector peptide, angiotensin II, has been related to the genesis and development of cardiovascular diseases (Barrios and Escobar 2008). Enalapril, an ACEi, is an orally administered prodrug that is hydrolyzed to the active form enalaprilat, which reduces the plasma levels of angiotensin II (Todd and Heel 1986). This reduction of angiotensin II results in peripheral vasodilatation and reduced vascular resistance, leading to a decrease in BP values (Hair et al 2007). Enalapril is a dose-dependent antihypertensive drug, with the maximum effect occurring after 6-8 hours of administration and a total effect duration of 24-36 hours (Todd and Heel 1986). Enalapril has shown to be an effective antihypertensive agent, with positive effects on cardiovascular risk factors and organ damage (Todd and Goa 1992; Ravid et al 1998, Hosomi et al 2001; Rosei et al 2005). Thus, enalapril reduced carotid artery intima-media thickness progression, an independent risk factor for cardiovascular and cerebrovascular disease (Hosomi et al 2001). Moreover, in a randomized, double-blind, 6-year trial in patients with diabetes and normoalbuminuria at baseline, enalapril reduced the development of microalbuminuria (Ravid et al 1998).

The combination of CCB and ACEi is especially effective due to their complementary mechanisms that enhance the antihypertensive efficacy with a low side effects rate (McInnes 2007). CCBs are potent vasodilators that induce reflex activation of the sympathetic system and RAAS. As a result, the use of an ACEi may buffer this excessive activation. Moreover, since $\mathrm{CCBs}$ promote a negative sodium balance and an increase of angiotensin II levels, this may reinforce the antihypertensive effect of ACEis (Gojanovic et al 2008). On the other hand, the concomitance of both treatments may reduce the presence of adverse events, mainly the peripheral oedema (Messerli 2002). Mechanistically, the development of lower extremity oedema during calcium entry blockade is due to an increase in intracapillary pressure, as a consequence of a selective diminution of the precapillary arteriolar tone. Interestingly, ACEis reduce the lower extremity oedema caused by CCBs, most likely because of their ability to dilate both the arterial vascular bed and the venous capacitance vessels (Weir et al 2001; Gojanovic et al 2008).

\section{Fixed combination lercanidipine plus enalapril: current evidence}

Several trials have demonstrated the benefits of the fixed combination of lercanidipine and enalapril (Recordati 2004a, b; Agrawal et al 2006; Puig et al 2007). In a trial performed in hypertensive patients who were nonresponders to lercanidipine, after 12 weeks of treatment with fixed combination lercanidipine plus enalapril, a significantly greater proportion of patients with this combination normalized their BP compared with patients treated with lercanidipine in monotherapy (22\% vs $12 \%, p=0.012$ ) (Recordati 2004a). Similarly, in another study developed in patients with hypertension who were nonresponders to enalapril, after 12 weeks of treatment with this fixed combination, there was a trend to a better BP control in the population treated with the fixed combination compared with enalapril in monotherapy (24\% vs $17 \%$, $\mathrm{p}=$ NS) (Recordati 2004b).

More recently, a double-blind, placebo-controlled, fourway balanced design crossover study included hypertensive patients aged 60-85 years with mean office-measured sitting systolic BP 160-179 mmHg and daytime ambulatory systolic BP $\geq 135$ mmHg (Puig et al 2007). After a 2-week run-in period, during which previous medications were discontinued, each patient were randomly allocated to lercanidipine $10 \mathrm{mg}$, enalapril $20 \mathrm{mg}$, lercanidipine $10 \mathrm{mg}$ plus enalapril $20 \mathrm{mg}$, or placebo. Of the 75 patients randomized, 62 completed the study. At study end, office BP was measured and a 24-hour ambulatory BP monitoring was performed. The administration of placebo, lercanidipine, enalapril, and lercanidipine plus enalapril was associated with a mean 24-hour systolic BP of 144, 137, 133, and $127 \mathrm{mmHg}$, respectively. All active treatments significantly reduced the mean 24-hour BP compared with placebo, but the combination lercanidipine plus enalapril was significantly more effective than the active components taken in monotherapy. Similarly, office systolic BP was significantly more reduced with the combination 
than with the monotherapies. A higher proportion of patients treated with the combination lercanidipine plus enalapril achieved BP goals compared with lercanidipine and enalapril alone ( $45 \%$ vs $18 \%$ vs $19 \%$, respectively).

The combination lercanidipine plus enalapril has been shown to be effective not only in the general hypertensive population but also in high-risk patients, such as those with diabetes. A controlled clinical trial investigated the efficacy of lercanidipine vs HCTZ as add-on therapy in diabetic patients with uncontrolled hypertension on enalapril monotherapy (Agrawal et al 2006). A total of 174 patients with well-controlled diabetes and mild to moderate hypertension were included in a 2-week placebo run-in followed by 4 weeks on enalapril $20 \mathrm{mg}$. Subsequently, 135 nonresponders were randomized to 20 weeks of double-blind add-on therapy to enalapril with either lercanidipine 10 $\mathrm{mg}(\mathrm{n}=69)$ or HCTZ $12.5 \mathrm{mg}(\mathrm{n}=66)$. BP response rates reached $69.6 \%$ on enalapril plus lercanidipine compared with $53.6 \%$ on enalapril plus HCTZ $(p=N S)$ and BP control was attained in $30.4 \%$ of patients on lercanidipine add-on and in $23.2 \%$ of those randomized to HCTZ add-on $(\mathrm{p}=\mathrm{NS})$. Figure 3 summarizes BP control rates achieved by the combination lercanidipine plus enalapril in different clinical trials.
The combination lercanidipine plus enalapril was well tolerated in all clinical trials, with a similar rate of side effects than the drugs in monotherapy (Recordati 2004a b; Agrawal et al 2006; Puig et al 2007). Although infrequent, the most prevalent adverse events related to the use of the combination lercanidipine plus enalapril were cough, dizziness, and vertigo. Interestingly, no clinically significant differences in heart rate were observed between groups and 24-hour heart rate remained stable under all treatments (Puig et al 2007). The absence of negative effects of the combination on lipid and glucose metabolism appears an added advantage in the treatment of hypertensive population (Hair et al 2007).

\section{Conclusions and future perspectives}

Most patients with hypertension often require more than one drug to achieve BP goals. The most recent European guidelines clearly establish that combination therapy is recommended as first-line therapy in certain situations, such as marked BP elevations, high or very high-risk patients, or when BP targets are lower (Mancia et al 2007). Clinical trials have shown that the combination lercanidipine plus enalapril is an effective and well-tolerated fixed-dose antihypertensive combination. As expected, it provides greater antihypertensive efficacy than either component taken as monotherapy.

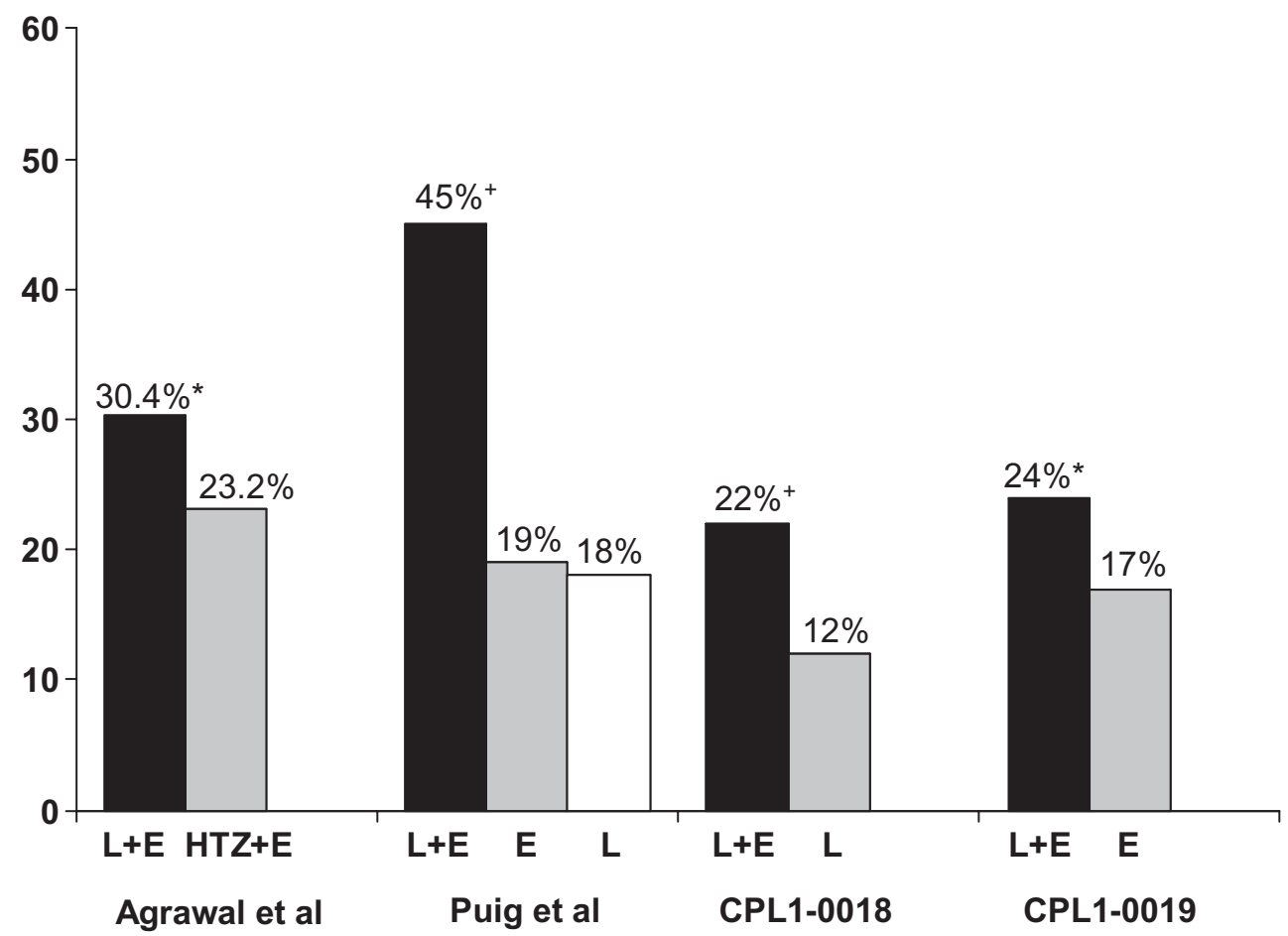

Figure 3 Blood pressure control rates achieved by the combination lercanidipine plus enalapril in different clinical trials (after Recordati 2004a, b; Agrawal et al 2006; Puig et al 2007). ${ }^{*} p=N S ;+p=0.01$.

Abbreviations: L, lercanidipine; E, enalapril; HTZ, hydrochlorothiazide. 
But to date studies assessing the effects of lercanidipine plus enalapril in reversing left ventricular hypertrophy or progression of renal disease, or improving clinical outcomes in hypertensive patients are still warranted .

\section{Disclosures}

None of the authors has any conflicts of interest to disclose.

\section{References}

Agrawal R, Marx A, Haller H. 2006. Efficacy and safety of lercanidipine versus hydrochlorothiazide as add-on to enalapril in diabetic populations with uncontrolled hypertension. J Hypertens, 24:185-92.

ALLHAT Officers and Coordinators for the ALLHAT Collaborative Research Group. 2002. Major outcomes in high-risk hypertensive patients randomized to angiotensin-converting enzyme inhibitor or calcium channel blocker vs diuretic: the Antihypertensive and Lipid-Lowering Treatment to Prevent Heart Attack Trial (ALLHAT). JAMA, 288:2981-97.

Bang LM, Chapman TM, Goa KL. 2003. Lercanidipine: a review of its efficacy in the management of hypertension. Drugs, 63:2449-72.

Bakris GL, Williams M, Dworkin L, et al. 2000. Preserving renal function in adults with hypertension and diabetes: a consensus approach. National Kidney Foundation Hypertension and Diabetes Executive Committees Working Group. Am J Kidney Dis, 36:646-61.

Barrios V, Navarro A, Esteras A, et al. 2002. Antihypertensive efficacy and tolerability of lercanidipine in daily clinical practice. The ELYPSE Study. Blood Press, 11:95-100.

Barrios V, Escobar C, Navarro A, et al. 2006. Lercanidipine is an effective and well tolerated antihypertensive drug regardless the cardiovascular risk profile: The LAURA study. Int J Clin Pract, 60:1364-70.

Barrios V, Banegas JR, Ruilope LM, et al. 2007a. Evolution of blood pressure control in Spain. J Hypertens, 25:1975-7.

Barrios V, Escobar C, Calderón A, et al. 2007b. CONTROLRISK Investigators. Cardiovascular risk profile and risk stratification of the hypertensive population attended by general practitioners and specialists in Spain. The CONTROLRISK study. J Hum Hypertens, 21:479-85.

Barrios V, Escobar C, Calderón A, et al. 2007c. Blood pressure and lipid goal attainment in the hypertensive population in the primary care setting in Spain. J Clin Hypertens (Greenwich), 9:324-9.

Barrios V, Escobar C, Calderón A, et al. 2007d. Prevalence of the metabolic syndrome in patients with hypertension treated in general practice in Spain: an assessment of blood pressure and low-density lipoprotein cholesterol control and accuracy of diagnosis. J Cardiometab Syndr, 2:9-15.

Barrios V, Escobar C, Llisterri JL, et al. 2007e. Blood pressure and lipid control and coronary risk in the hypertensive population attended in Primary Care setting in Spain. The PRESCOT study. Rev Clin Esp, 207:172-8.

Barrios V, Escobar C. 2008. Olmesartan medoxomil plus hydrochlorothiazide for treating hypertension. Expert Opin Pharmacother, 9:129-36.

Barrios V, Escobar C, de la Figuera M, et al. 2008. High doses of lercanidipine are better tolerated than other dihydropyridines in hypertensive patients with metabolic syndrome: results from the TOLERANCE study. Int J Clin Pract, 62:723-8.

Brenner BM, Cooper ME, de Zeeuw D, et al. 2001. Effects of losartan on renal and cardiovascular outcomes in patients with type 2 diabetes and nephropathy. N Engl J Med, 345:861-9.

Canavesi M, Baldini N, Leonardi A, et al. 2004. In vitro inhibitory effect of lercanidipine on cholesterol accumulation and matrix metalloproteinases secretion by macrophages. J Cardiovasc Pharmacol, 44:416-22.

Cherubini A, Fabris F, Ferrari E, et al. 2003. Comparative effects of lercanidipine, lacidipine, and nifedipine gastrointestinal therapeutic system on blood pressure and heart rate in elderly hypertensive patients: the ELderly and LErcanidipine (ELLE) study. Arch Gerontol Geriatr, $37: 203-12$.
Cheung BM, Ong KL, Man YB, et al. 2006. Prevalence, awareness, treatment, and control of hypertension: United States National Health and Nutrition Examination Survey 2001-2002. J Clin Hypertens (Greenwich), 8:93-8

Chobanian AV, Bakris GL, Black HR, et al. 2003. The Seventh Report of the Joint National Committee on Prevention, Detection, Evaluation, and Treatment of High Blood Pressure: the JNC 7 report. JAMA, 289:2560-72.

Dahlöf B, Sever PS, Poulter NR, et al. 2005. Prevention of cardiovascular events with an antihypertensive regimen of amlodipine adding perindopril as required versus atenolol adding bendroflumethiazide as required, in the Anglo-Scandinavian Cardiac Outcomes Trial-Blood Pressure Lowering Arm (ASCOT-BPLA): a multicentre randomised controlled trial. Lancet, 366:895-906.

Dalla Vestra M, Pozza G, Mosca A, et al. 2004. Effect of lercanidipine compared with ramipril on albumin excretion rate in hypertensive Type 2 diabetic patients with microalbuminuria: DIAL study (diabete, ipertensione, albuminuria, lercanidipina). Diabetes Nutr Metab, 17:259-66.

Egan BM. 2007. Combination therapy with an angiotensin-converting enzyme inhibitor and a calcium channel blocker. J Clin Hypertens (Greenwich), 9:783-89.

Epstein M. 2001. Lercanidipine: a novel dihydropyridine calcium-channel blocker. Heart Dis, 3:398-407.

Estacio RO, Schrier RW. 1998. Antihypertensive therapy in type 2 diabetes: implications of the appropriate blood pressure control in diabetes (ABCD) trial. Am J Cardiol, 82:9R-14R.

Fogari R, Corradi L, Zoppi A, et al. 2007a. Addition of manidipine improves the antiproteinuric effect of candesartan in hypertensive patients with type II diabetes and microalbuminuria. Am J Hypertens, 20:1092-96.

Fogari R, Derosa G, Zoppi A, et al. 2007b. Effect of telmisartan-amlodipine combination at different doses on urinary albumin excretion in hypertensive diabetic patients with microalbuminuria. Am J Hypertens, 20:417-22.

Fogari R, Malamani G, Zoppi A, et al. 2007c. Effect on the development of ankle edema of adding delapril to manidipine in patients with mild to moderate essential hypertension: a three-way crossover study. Clin Ther, 29:413-18.

Gojanovic B, Feihl F, Liaudet L, et al. 2008. Concomitant calcium entry blockade and inhibition of the renin-angiotensin system: a rational and effective means for treating hypertension. $J$ Renin Angiotensin Aldosterone Syst, 9:1-9.

Hair PI, Scott LJ, Perry CM. 2007. Fixed-dose combination lercanidipine/ enalapril. Drugs, 67:95-106.

Hansson L, Zanchetti A, Carruthers SG, et al. 1998. Effects of intensive blood-pressure lowering and low-dose aspirin in patients with hypertension: principal results of the Hypertension Optimal Treatment (HOT) randomised trial. HOT Study Group. Lancet, 351:1755-62.

Hasebe N, Kikuchi K; NICE Combi Study Group. 2005. Controlled-release nifedipine and candesartan low-dose combination therapy in patients with essential hypertension: the NICE Combi (Nifedipine and Candesartan Combination) Study. J Hypertens, 23:445-53.

Herbette LG, Vecchiarelli M, Sartani A, et al. 1998. Lercanidipine: short plasma half-life, long duration of action and high cholesterol tolerance. Updated molecular model to rationalize its pharmacokinetic properties. Blood Press Suppl, 2:10-17.

Hosomi N, Mizushige K, Ohyama H, et al. 2001. Angiotensin-converting enzyme inhibition with enalapril slows progressive intima-media thickening of the common carotid artery in patients with non-insulindependent diabetes mellitus. Stroke, 32:1539-45.

James IG, Jones A, Davies P. 2002. A randomised, double-blind, doubledummy comparison of the efficacy and tolerability of lercanidipine tablets and losartan tablets in patients with mild to moderate essential hypertension. J Hum Hypertens, 16:605-10.

Julius S, Kjeldsen SE, Brunner H, et al. 2003. VALUE trial: Long-term blood pressure trends in 13,449 patients with hypertension and high cardiovascular risk. Am J Hypertens, 16:544-8. 
Lazarus JM, Bourgoignie JJ, Buckalew VM, et al. 1997. Achievement and safety of a low blood pressure goal in chronic renal disease. The Modification of Diet in Renal Disease Study Group. Hypertension, 29:641-50.

Leonetti G, Magnani B, Pessina AC, et al. 2002. Tolerability of long-term treatment with lercanidipine versus amlodipine and lacidipine in elderly hypertensives. Am J Hypertens, 15:932-40.

Lewis EJ, Hunsicker LG, Clarke WR, et al. 2001. Renoprotective effect of the angiotensin-receptor antagonist irbesartan in patients with nephropathy due to type 2 diabetes. $N$ Engl J Med, 345:851-60.

Liu L, Wang JG, Gong L, et al. 1998. Comparison of active treatment and placebo in older Chinese patients with isolated systolic hypertension. Systolic Hypertension in China (Syst-China) Collaborative Group. J Hypertens, 16:1823-29.

McInnes GT. 2007. Antihypertensive drugs in combination: additive or greater than additive? J Hum Hypertens, 21:914-16.

Mancia G, De Backer G, Dominiczak A, et al. 2007. 2007 Guidelines for the Management of Arterial Hypertension: The Task Force for the Management of Arterial Hypertension of the European Society of Hypertension (ESH) and of the European Society of Cardiology (ESC). J Hypertens, 25:1105-87.

Meredith PA. 1999. Lercadinidipine: a novel lipophilic dihydropyridine calcium antagonist with long duration of action and high vascular selectivity. Exp Opin Invest Drugs, 8:1043-62.

Messerli FH. 2002. Vasodilatory edema: a common side effect of antihypertensive therapy. Curr Cardiol Reports, 4:479-82.

Motwani JG. 2002. Combining renin-angiotensin-aldosterone system blockade with diuretic therapy for treatment of hypertension. $J$ Renin Angiotensin Aldosterone Syst, 3:72-78.

Nesbitt SD. 2007. Antihypertensive combination therapy: optimizing blood pressure control and cardiovascular risk reduction. J Clin Hypertens (Greenwich), 9(11 Suppl 4):26-32.

Nissen SE, Tuzcu EM, Libby P, et al. 2004. Effect of antihypertensive agents on cardiovascular events in patients with coronary disease and normal blood pressure: the CAMELOT study: a randomized controlled trial. JAMA, 292:2217-25.

Ong KL, Cheung BM, Man YB, et al. 2007. Prevalence, awareness, treatment, and control of hypertension among United States adults 1999-2004. Hypertension, 49:69-75.

Otero ML. 2007. Manidipine-delapril combination in the management of hypertension. Vasc Health Risk Manag, 3:255-63.

Poldermans D, Glazes R, Kargiannis S, et al. 2007. Tolerability and blood pressure-lowering efficacy of the combination of amlodipine plus valsartan compared with lisinopril plus hydrochlorothiazide in adult patients with stage 2 hypertension. Clin Ther, 29:279-89.

Puig JG, Calvo C, Luurila O, et al. 2007. Lercanidipine, enalapril and their combination in the treatment of elderly hypertensive patients: placebo-controlled, randomized, crossover study with four ABPM. J Hum Hypertens, 21:917-24.

Rachmani R, Levi Z, Zadok BS, et al. 2002. Losartan and lercanidipine attenuate low-density lipoprotein oxidation in patients with hypertension and type 2 diabetes mellitus: a randomized, prospective crossover study. Clin Pharmacol Ther, 72:302-7.

Ravid M, Brosh D, Levi Z, et al. 1998. Use of enalapril to attenuate decline in renal function in normotensive, normoalbuminuric patients with type 2 diabetes mellitus. A randomized, controlled trial. Ann Intern Med, 128:982-8.
Recordati SpA. 2004a. Efficacy and tolerability of a combination of lercanidipine and enalapril in patients with mild to moderate essential hypertension not adequately controlled by lercanidipine treatment (add-on to lercanidipine) [CPL1-0018]. Milan: Recordati SpA, Mar 11. [Data on file].

Recordati SpA. 2004b. Efficacy and tolerability of a combination of lercanidipine and enalapril in patients with mild to moderate essential hypertension not adequately controlled by enalapril treatment (add-on to enalapril) [CPL1-0019]. Milan: Recordati SpA, Mar 25. [Data on file].

Robles NR, Ocon J, Gomez CF, et al. 2005. Lercanidipine in patients with chronic renal failure: the ZAFRA study. Ren Fail, 27:73-80.

Robles NR, Pastor L, Manjón M, et al. 2004. Lercanidipine in diabetic patients with renal failure. Nefrologia, 24:338-43.

Romito R, Pansini MI, Perticone F, et al. 2003. Comparative effect of lercanidipine, felodipine, and nifedipine GITS on blood pressure and heart rate in patients with mild to moderate arterial hypertension: the Lercanidipine in Adults (LEAD) Study. J Clin Hypertens (Greenwich), 5:249-53.

Rosei EA, Rizzoni D, Muiesan ML, et al. 2005. Effects of candesartan cilexetil and enalapril on inflammatory markers of atherosclerosis in hypertensive patients with non-insulin-dependent diabetes mellitus. J Hypertens, 23:435-44.

Ryden L, Standl E, Bartnik M, et al. 2007. Guidelines on diabetes, prediabetes, and cardiovascular diseases: Executive summary. The Task Force on Diabetes and Cardiovascular Diseases of the European Society of Cardiology (ESC) and of the European Association for the Study of Diabetes (EASD). Eur Heart J, 28:88-136.

Sica DA. 2002. Rationale for fixed-dose combinations in the treatment of hypertension: the cycle repeats. Drugs, 62:44-62.

Soma MR, Natali M, Donetti E, et al. 1998. Effect of lercanidipine and its (R)-enantiomer on atherosclerotic lesions induced in hypercholesterolemic rabbits. Br J Pharmacol, 125:1471-6.

Sowers JR, Bakris GL, Black HR, et al. 2007. The cardiometabolic syndrome and calcium channel blocker combination drugs. J Cardiometab Syndr, 2:207-12.

Staessen JA, Fagard R, Thijs L, et al. 1997. Randomised double-blind comparison of placebo and active treatment for older patients with isolated systolic hypertension. The Systolic Hypertension in Europe (Syst-Eur) Trial Investigators. Lancet, 350:757-64.

Todd PA, Goa KL. 1992. Enalapril. A reappraisal of its pharmacology and therapeutic use in hypertension. Drugs, 43:346-81.

Todd PA, Heel RC. Enalapril. 1986. A review of its pharmacodynamic and pharmacokinetic properties, and therapeutic use in hypertension and congestive heart failure. Drugs, 31:198-248.

UKPDS 38. 1998. Tight blood pressure control and risk of macrovascular and microvascular complications in type 2 diabetes: UKPDS 38. UK Prospective Diabetes Study Group. BMJ, 317:703-13.

Wang YR, Alexander GC, Stafford RS. 2007. Outpatient hypertension treatment, treatment intensification, and control in Western Europe and the United States. Arch Intern Med, 167:141-7.

Weir MR, Rosenberger C, Fink JC. 2001. Pilot study to evaluate a water displacement technique to compare effects of diuretics and ACE inhibitors to alleviate lower extremity edema due to dihydropyridine calcium antagonists. Am J Hypertens, 14:963-8. 
